Acta Chemica Scandinavica A 33 (1979) 583-592

\title{
Brønsted's Principle of Specific Interaction of Ions and the Mean Ionic Activity Coefficients in Aqueous Solutions of Earth Alkali Halogenides. Calculation of Cationic Radii from the ASPEV
} Theory

\author{
TORBEN SMITH SØRENSEN *
}

Fysisk Laboratorium III, Technical University of Denmark, DK-2800 Lyngby, Denmark

The adjusted screened potential/excluded volume theory (ASPEV) is used to fit mean ionic activity coefficients as functions of concentrations for all combinations of the ions $\mathrm{Mg}^{2+}, \mathrm{Ca}^{2+}, \mathrm{Sr}^{2+}, \mathrm{Ba}^{2+}$ with the ions $\mathrm{Cl}^{-}, \mathrm{Br}^{-}, \mathrm{I}^{-}$. The formulae derived may be used up to $5-6 \mathrm{~mol} / \mathrm{dm}^{3}$ (ionic strengths $15-18$ ) with good accuracy.

From the second virial coefficients, radii of the solvated cations can be calculated from the basic assumptions that (1) the Brønsted principle of specific interaction of ions is valid and (2) the anions are unsolvated. The radii of the earth alkali metal cations can-together with the radii of the alkali metal cations previously found and the Pauling radii of the halogenide ions - be related to the equivalent ionic conductivities by means of a modified Born-Fuoss-Boyd-Zwanzig theory of dielectric relaxation drag in addition to the Stokes' law viscous drag.

The ASPEV radii of the alkali metal ions and the earth alkali metal ions can be plotted on a common curve $v s$. the surface density of squared charge on the "Pauling surface" of the ions. This is explained to be due to orientation of the water molecules by the electric field strength and the ion-dipole interaction. When an ion becomes smaller in magnitude than the "holes" in the water structure, a further increase in surface density of squared charge has no further effect in binding water and the ASPEV radius remains constant at a saturation value.

\footnotetext{
* On leave from Fysisk-Kemisk Institut, Technical University of Denmark.
}

The Adjusted Screened Potential/Excluded Volume (ASPEV) model for calculation of ionic activity coefficients has been discussed in a previous publication. ${ }^{1}$ The basic idea is to make an analogy with the virial expansion of the pressure in a mixture of hard-sphere gases and the virial expansion of the osmotic pressure in electrolyte solutions. The interaction between the Debye-Hückel terms (of the order $3 / 2$ in the concentration) and the excluded volume terms (of the order 2 in the concentration) makes some semi-empirical additional assumptions necessary, however. First, it is assumed that excluded volumes between ions of the same sign can be neglected in comparison with excluded volumes between ions of opposite signs due to the electrostatic repulsion between ions with charges of the same sign. This assumption may be seen as a quantification of the principle of specific interaction of ions put forward by Brønsted (though in a somewhat unclear fashion) in $1921 .^{2}$ The interpretation of Brønsted's principle used in the ASPEV theory is similar to the interpretation common in modern electrochemical textbooks, ${ }^{3}$ although no authors have hitherto attempted calculations of ionic radii from such data. The second assumption in the ASPEV theory is the following: Since an ion already at an ionic strength around $0.001 \mathrm{~mol} / \mathrm{dm}^{3}$ is surrounded by an "ionic cloud" of counter ions, the "effective charges" with which the ions interact electrostatically are less than their real charges. This screening effect tends to lower the numerical contribution of the term in the expression for the activity coefficients proportional to the square root 
of the ionic strength. Starting "from behind" (from the high salt concentrations) it is possible in some instances to find empirical formulae relating the screening effect to the ionic strength and to the excluded volume between anion and cation. ${ }^{1}$

Although Brønsted's principle is normally invoked only for the case of multi-electrolyte mixtures, it may equally well be applied to the case of only a single electrolyte in solution. When this was done for the alkali metal halogenides ${ }^{1}$ cationic radii were found which were well in accordance with radii estimated from equivalent ionic conductivities and the Born-Fuoss-Boyd-Zwanzig theory of dielectric relaxation drag. The purpose of the present paper is to apply the ASPEV-procedure to activity coefficient data for all combinations of the ions $\mathrm{Mg}^{2+}, \mathrm{Ca}^{2+}, \mathrm{Sr}^{2+}, \mathrm{Ba}^{2+}$ and $\mathrm{Cl}^{-}, \mathrm{Br}^{-}, \mathrm{I}^{-}$. In particular it will be shown that the Brønsted principle gives consistent radii of the hydrated cations, when the anions are assumed unhydrated. The ion $\mathrm{Be}^{2+}$ is excluded from the above scheme only because of lack of data. The same is the case for the fluorides, which are mostly very sparingly soluble.

\section{TREATMENT OF CURVES OF ACTIVITY COEFFICIENTS VERSUS IONIC STRENGTH}

The data for the activity coefficients (mean ionic) of the earth alkali halogenides were taken from Refs. 4, 5 and 6. Those data give the molal activity coefficients versus the molality, but according to the ASPEV theory the calculable entities are the molar activity coefficients as a function of the molar concentration. Molar activity coefficients are calculated from the molal activity coefficients by means of the formula

$$
f_{ \pm}=\gamma_{ \pm} /(c / m)
$$

The density data necessary to find $c / m$ as a function of molality $m$ were taken from Ref. 7 and $c / m v s . m$ was in each case fitted by the statistically most significant polynomium. For approximate purposes, however, the following linear relation may be used

$c / m=0.997-K m\left(25^{\circ} \mathrm{C}\right)$

where the slope parameter $K$ for each electrolyte was averaged over the entire concentration range given in Ref. 7. For the vector of electrolytes $\left(\mathrm{MgCl}_{2}, \mathrm{MgBr}_{2}, \mathrm{MgI}_{2}, \mathrm{CaCl}_{2}, \mathrm{CaBr}_{2}, \mathrm{CaI}_{2}, \mathrm{SrCl}_{2}\right.$, $\mathrm{SrBr}_{2}, \mathrm{SrI}_{2}, \mathrm{BaCl}_{2}, \mathrm{BaBr}_{2}, \mathrm{BaI}_{2}$ ) we obtain the

Table 1. Characteristics and optimal values of $A^{*}$ and $B^{*}$ for $\ln f_{ \pm} v s . x$ data for earth alkali halogenides. ${ }^{a}$

\begin{tabular}{|c|c|c|c|c|c|c|c|c|}
\hline Electrolyte & $\begin{array}{l}x_{\min } \\
\left(\mathrm{mol} / \mathrm{dm}^{3}\right)^{\frac{1}{2}}\end{array}$ & $\left(\ln f_{ \pm}\right)_{\min }$ & $\begin{array}{l}x_{1 / 2} \\
\left(\mathrm{~mol} / \mathrm{dm}^{3}\right)^{\frac{1}{2}}\end{array}$ & $\begin{array}{l}x_{0} \\
\left(\mathrm{~mol} / \mathrm{dm}^{3}\right)^{\frac{1}{2}}\end{array}$ & $\begin{array}{l}A_{\mathrm{opl}}^{*} \\
\left(\mathrm{dm}^{3} / \mathrm{mol}\right)^{\frac{1}{2}}\end{array}$ & $A_{\text {regr. }}^{*}$ & $\begin{array}{l}B_{\mathrm{opt}}^{*} \\
\mathrm{dm}^{3} / \mathrm{mol}\end{array}$ & Ref. \\
\hline $\mathrm{MgCl}_{2}{ }^{*}$ & 0.97 & -0.670 & 1.90 & 2.25 & 1.20 & 1.24 & 0.53 & 5 (first set) \\
\hline $\mathrm{MgCl}_{2}$ & 1.00 & -0.738 & 1.95 & $2.31_{5}$ & 1.28 & 1.25 & 0.55 & \\
\hline $\mathrm{MgBr}_{3}{ }^{*}$ & 0.87 & -0.595 & 1.62 & 1.93 & 1.24 & 1.29 & 0.64 & 5 (first set) \\
\hline $\mathrm{MgBr}_{2}$ & 0.84 & -0.664 & 1.66 & $1.99_{5}$ & 1.34 & 1.31 & 0.68 & \\
\hline $\mathrm{MgI}_{2}{ }^{*}$ & 0.77 & -0.538 & 1.44 & 1.70 & 1.28 & 1.33 & 0.75 & 5 (first set) \\
\hline $\mathrm{MgI}_{2}$ & 0.76 & -0.572 & $1.47_{5}$ & $1.75_{5}$ & 1.32 & 1.34 & 0.76 & 6 \\
\hline $\mathrm{CaCl}_{2}$ & 1.10 & -0.792 & 2.15 & $2.55^{\circ}$ & 1.22 & 1.22 & 0.48 & 4,6 \\
\hline $\mathrm{CaBr}_{2}$ & 1.00 & -0.720 & 1.86 & 2.21 & 1.30 & 1.27 & 0.60 & 6 \\
\hline $\mathrm{CaI}_{2}$ & 0.82 & -0.634 & 1.62 & 1.93 & 1.32 & 1.31 & 0.69 & 6 \\
\hline $\mathrm{SrCl}_{2}$ & 1.19 & -0.822 & $2.29_{5}$ & 2.72 & 1.22 & 1.21 & 0.45 & 6 \\
\hline $\mathrm{SrBr}_{2}$ & 1.04 & -0.750 & 2.00 & 2.36 & 1.28 & 1.25 & 0.54 & 6 \\
\hline $\mathrm{SrI}_{2}$ & 0.86 & -0.650 & 1.69 & 2.02 & 1.30 & 1.29 & 0.65 & 6 \\
\hline $\mathrm{BaCl}_{2}$ & 1.38 & 0.936 & $x_{3 / 4}=2.34$ & - & 1.20 & 1.19 & 0.39 & 4,5 \\
\hline $\mathrm{BaCl}_{2} *$ & 1.36 & -0.900 & $x_{3 / 4}=2.40$ & - & 1.12 & 1.17 & 0.34 & 6 \\
\hline $\mathrm{BaBr}_{2}$ & 1.13 & -0.802 & 2.22 & $(2.64)$ & 1.22 & 1.22 & 0.46 . & 6 \\
\hline $\mathrm{BaI}_{2}$ & 0.89 & -0.680 & $1.72_{5}$ & 2.07 & 1.32 & 1.29 & $0.65^{\circ}$ & 6 \\
\hline
\end{tabular}

\footnotetext{
${ }^{a}$ It is suggested that the data with an asterisk $\left(^{*}\right)$ be left out due to the lack of correspondence between $A_{\mathrm{opt}}^{*}$ and $A_{\text {regr. }}^{*}$ shown in those cases.
} 
following vector of $K$-values at $25^{\circ} \mathrm{C}:(0.023,0.035$, $0.046,0.026,0.036,0.051,0.026,0.039,0.053,0.030$, $0.046,0.059$ ). Molar concentrations in $\mathrm{mol} / \mathrm{dm}^{3}$ and molal concentrations in $\mathrm{mol} / \mathrm{kg}$ water. Strictly speaking the data for the densities as function of the weight percentage of salt given in Ref. 7 are valid at a temperature of $20{ }^{\circ} \mathrm{C}$ and not $25{ }^{\circ} \mathrm{C}$. However, we have simply multiplied the densities with the ratio $\rho_{25} / \rho_{20}$ for pure water in order to have an approximation for the densities at $25^{\circ} \mathrm{C}$. It has been checked that the $c / m$ curve thus obtained for $\mathrm{BaCl}_{2}$ is practically identical to the curve obtained from the data for $\mathrm{BaCl}_{2}$ in Ref. 8 at $25^{\circ} \mathrm{C}$.

After the transformation from molal mean ionic activity coefficients to molar activity coefficients and transformation of molalities to molarities, $\ln f_{ \pm}$for the earth alkali halogenides were plotted against molar ionic strength $(I)$. For 2:1 electrolytes we have

$I=3 c$

where $c$ is the salt concentration. We now postulate an expression of the form

$\ln f_{ \pm}=-A^{*} \sqrt{I}+B^{*} I=-A^{*} x+B^{*} x^{2}$

with $x$ for $\sqrt{I}$. The characteristics chosen here to characterize the curves are $x_{\min },\left(\ln f_{ \pm}\right)_{\min }, x_{1 / 2}$ and $x_{0}$. Those are the $x$-value of the activity minimum, the depth of the minimum, the halfminimum value of $x$ to the right of the minimum and the pseudoideal point. For an illustration vide Ref. 1, Fig. 3. For given values of the characteristics, the assump-

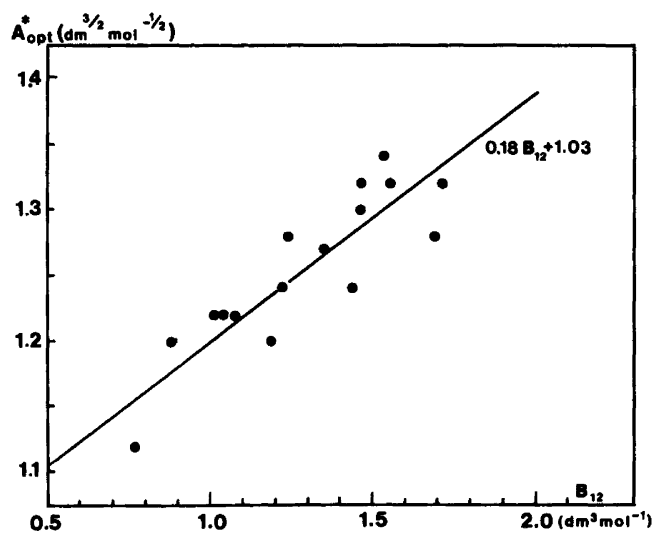

Fig. 1. The optimal $A^{*}$-values os. excluded volumes $B_{12}$ calculated from optimal $B^{*}$-values. Activity coefficient data for all combinations of $\mathrm{Mg}, \mathrm{Ca}$, $\mathrm{Sr}, \mathrm{Ba}$ with $\mathrm{Cl}, \mathrm{Br}$ and $\mathrm{I}$.

tion of true parabolic dependence of $\ln f_{ \pm}$on $x$ leads to a relation between $A^{*}$ and $B^{*}$ for each of the characteristics, see Ref. 1 , eqns. $(68 \mathrm{a}-\mathrm{e})$ and Fig. 4. Just as the case was for the alkali halogenides, it is possible in the present case to select optimal values of $A^{*}$ and $B^{*}$ which give simultaneously the right values of $\ln f_{ \pm}, x_{1 / 2}$ and $x_{0}$ (but not the $x_{\min }$ values). In Table 1 the values of the characteristics are listed together with the optimal $A^{*}$ and $B^{*}$ values for each electrolyte studied. In some cases the differences between the various literature data for the activity coefficients are so large that it is impossible to know a priori which are the best ones. In such cases both sets of data were analyzed parallel to each other.

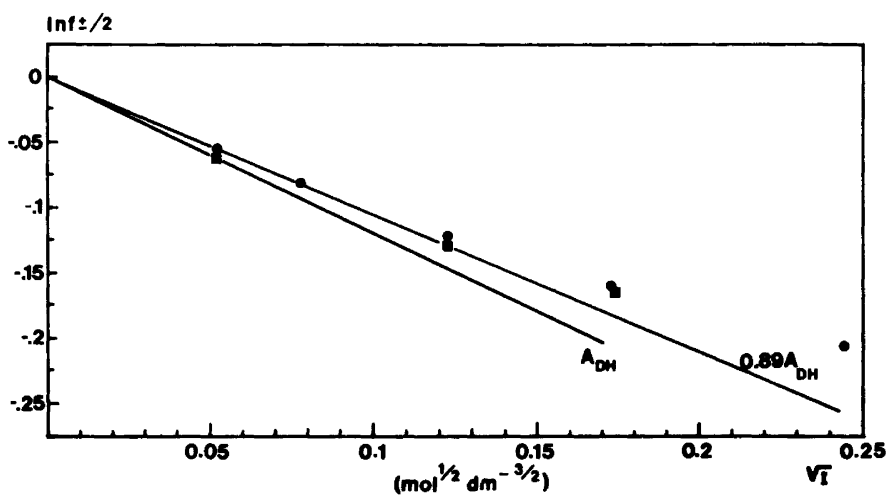

Fig. 2. $\ln f_{ \pm}$vs. $\sqrt{I}$ for small ionic strengths for $\mathrm{CaCl}_{2}(\bullet)$ and $\mathrm{BaCl}_{2}(\mathbf{\square})$ in water at $25^{\circ} \mathrm{C}$.

Acta Chem. Scand. A 33 (1979) No. 8 
As in the case of the alkali halogenides there seems to be a clearcut correlation between $A_{\mathrm{opt}}^{*}$ and $B_{\mathrm{opt}}^{*}$. This is shown in Fig. 1 where $A_{\mathrm{opt}}^{*}$ is plotted $v s . B_{12}=(9 / 4) B_{\mathrm{opt}}^{*}$. The reason for the factor $(9 / 4)$ will be given later on. The regression line is the following:

$$
A_{\text {regr. }}^{*}=0.18 B_{12}+1.03\left(B_{12}=\frac{9}{4} B_{\text {opt }}^{*}\right)
$$

In Table 1 the values of $A_{\text {regr. }}^{*}$ calculated from (5) are also given for comparison with the directly found $A_{\mathrm{opt}}^{*}$ - values. The deviation is seldom more than 0.03 . Luckily, the most deviating cases (marked with an asterisk in the table) occur for one data-set out of the 4 double valued data-sets in the table. Therefore, I shall make short work of those data and leave them out - hoping not to commit an injustice.

Data in the concentration range 0.001 to 0.1 $\mathrm{mol} / \mathrm{dm}^{3}$ are unfortunately only found for $\mathrm{CaCl}_{2}$ and $\mathrm{BaCl}_{2}$ (in Ref. 4). In Fig. 2 it is seen that a straight line with slope $-2 A_{\mathrm{DH}}$ (with a Debye-Hückel slope $A_{\mathrm{DH}}=1.178$ ) is a little too steep to fit the first part of the data. As for the alkali halogenides the slope $0.89 \times 2 A_{\mathrm{DH}}$ yields a better fit. Unhappily, in Ref. 1 Fig. 2, the Debye-Hückel line was too steeply drawn, so it seemed that the limiting law was much worse than it actually is. The other straight lines in this figure are correctly drawn, however, and the error committed has no effect on the calculations in Ref. 1.

The variation of the $A^{*}$ coefficient with ionic strength in the region to the left of the activity coefficient minimum is now found from subtraction

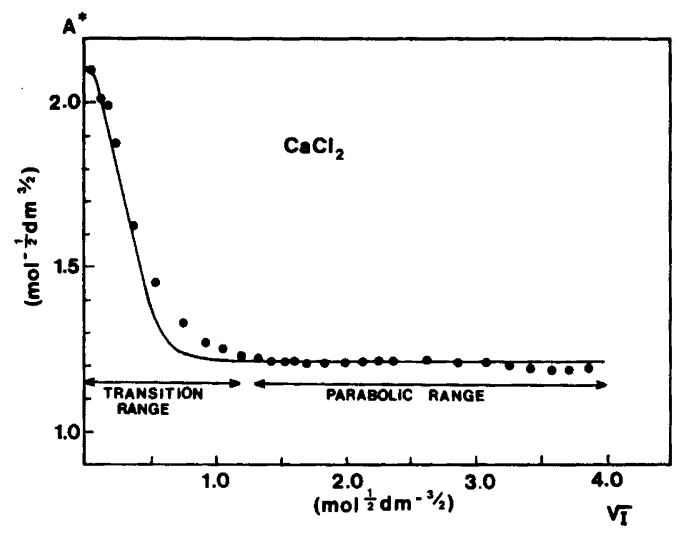

Fig. 3. $A^{*}$ vs. $\sqrt{I}$ for $\mathrm{CaCl}$.

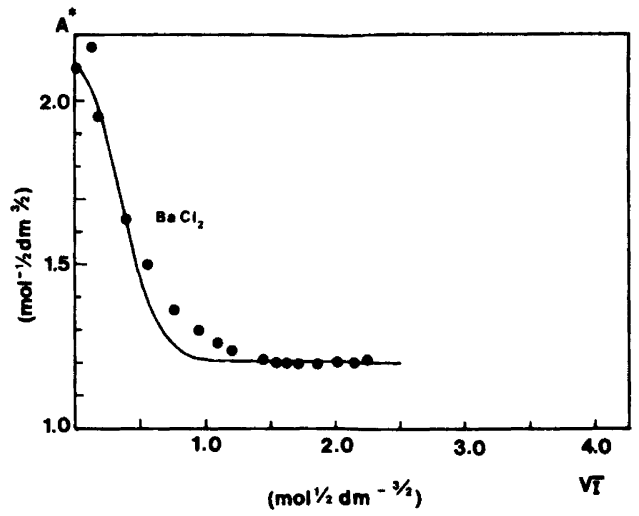

Fig. 4. $A^{*}$ vs. $\sqrt{I}$ for $\mathrm{BaCl}_{2}$.

$A^{*}=-\frac{\ln f_{ \pm}}{\sqrt{I}}+B_{\mathrm{opt}}^{*} \sqrt{I}$

for the two cases $\mathrm{CaCl}_{2}$ and $\mathrm{BaCl}_{2}$. The results are shown in Figs. 3 and 4. Especially Fig. 3 for $\mathrm{CaCl}_{2}$, where measurements extend up to $6 \mathrm{~mol} / \mathrm{kg}$ water, furnishes a strong case for "starting from behind" (great concentrations) in the analysis, since the $\ln f_{ \pm} v s . x$ curve is really a parabola (constant $A^{*}$ ) in a substantial concentration range.

The full lines drawn in Figs. 3 and 4 correspond to the expression

$A^{*}=A_{\mathrm{opt}}^{*}+\left(2 \times 0.89 A_{\mathrm{DH}}-A_{\mathrm{opt}}^{*}\right) \mathrm{e}^{-6 B_{12} l}$

and it is seen that they are not inconsistent with the data. A better fit might be obtained by adjusting the exponential factor, but this is hardly interesting for the following reason: In the case of the alkali halogenides we found a similar expression as (7), but with $\exp (-18 B I)$. In the case of $1: 1$ electrolytes $B^{*}$ is directly equal to $B_{12}$, i.e. the excluded volume between the anion and cation in $\mathrm{dm}^{3}$ per mol, see eqn. (23) in Ref. 1. It is fair to assume that this is the relevant parameter also in the case of 2:1 electrolytes to be taken in the exponential function. Also, salt concentration and ionic strength coincide for 1:1 electrolytes. Assuming that the ionic strength dependence is already taken into account by the square root of $I$ multiplying $A^{*}$, the exponential decay is determined by space restrictions, and the fundamental dimensionless parameter is $B_{12} c$ rather than $B_{12} I$. Since $I$ for $2: 1$ electrolytes is equal to three times the concentration, we recover in (7) 
the factor 18 in the argument of the exponential, when $I$ is replaced by $3 c$.

If one wishes, the expression for the activity coefficients in water at $25{ }^{\circ} \mathrm{C}$ can be expressed in terms of two non-dimensional numbers. The first number is a non-dimensional concentration

$c^{\prime}=B_{12} c$

and the second a non-dimensional excluded volume

$B_{12}^{\prime}=55.5 B_{12}$

In the latter case non-dimensionalization is made by means of the molar concentration of pure water. Such a non-dimensional excluded volume might be of interest in comparing electrolyte solutions in different solvents. The final expression is the following:

$\ln f_{ \pm}=-A^{\prime} \sqrt{3 c^{\prime}}+\frac{4}{3} c^{\prime}$

The non-dimensional parameter $A^{\prime}$ is given by

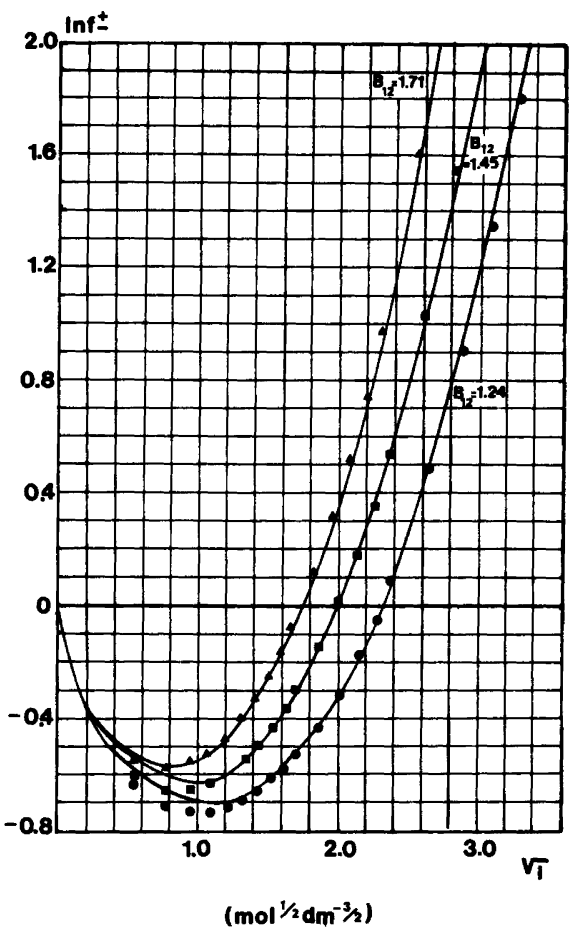

Fig. 5. Mean (molar) ionic activity coefficients $v s$. $\sqrt{I}$ for $\mathrm{MgCl}_{2}(\bullet), \mathrm{MgBr}_{2}(\boldsymbol{\square})$ and $\mathrm{MgI}_{2}(\boldsymbol{\Delta})$ in water at $25^{\circ} \mathrm{C}$. Full lines: ASPEV theory with $B_{12}$ in $\mathrm{dm}^{3} / \mathrm{mol}$.

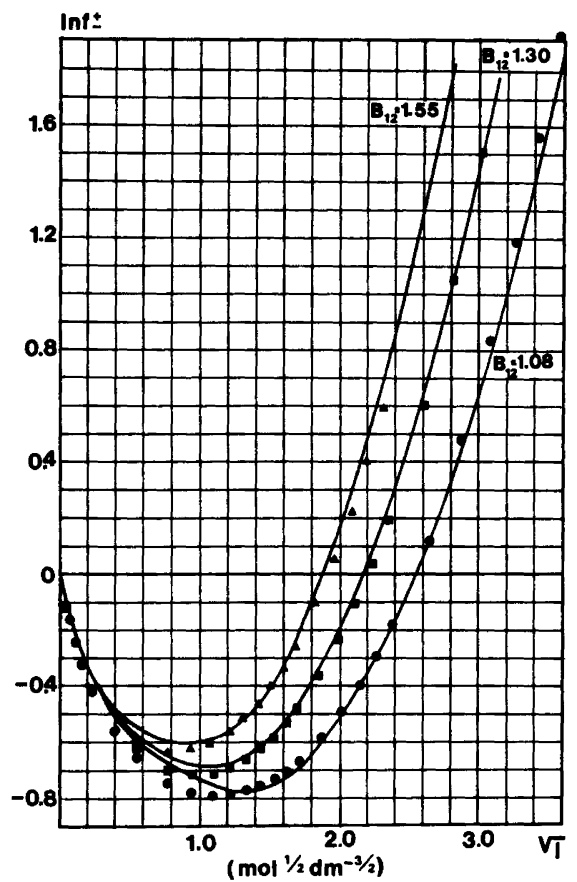

Fig. 6. $\ln f_{ \pm}$vs. $\sqrt{I}$ for $\mathrm{CaCl}_{2}(\bullet), \mathrm{CaBr}_{2}(\mathbf{\square})$ and $\mathrm{CaI}_{2}(\boldsymbol{\Lambda})$. Full lines: ASPEV.

$A^{\prime}=\frac{1}{\sqrt{B_{12}^{\prime}}}\left[7.68+0.0242 B_{12}^{\prime}+\right.$

$\left.\left(7.97-0.0242 B_{12}^{\prime}\right) e^{-18 c^{\prime}}\right]$

The formulae $(10-11)$ are valid for 2:1 electrolytes (or at least the investigated earth alkali halogenides) in water at $25^{\circ} \mathrm{C}$. For $1: 1$ electrolytes (or at least alkali halogenides) in water at $25^{\circ} \mathrm{C}$ we obtain from Ref. 1 the analogous equations

$\ln f_{ \pm}=-A^{\prime} \sqrt{c^{\prime}}+c^{\prime}$

$A^{\prime}=\frac{1}{\sqrt{B_{12}^{\prime}}}\left[4.97+0.0332 B_{12}^{\prime}+\right.$

$\left.\left(2.85-0.0332 B_{12}^{\prime}\right) \mathrm{e}^{-18 c^{\prime}}\right]$

The results of the fitting procedure for the activity coefficients of the earth alkali halogenides are shown in Figs. 5-8. The fit is seen to be quite good even up to such high concentrations as $6 \mathrm{~mol} / \mathrm{dm}^{3}$. Since the values of $A^{*}$ are taken as the regression values and not the optimal values, slight systematic deviations will appear at the high concentrations for some of the electrolytes. In the worst cases the 


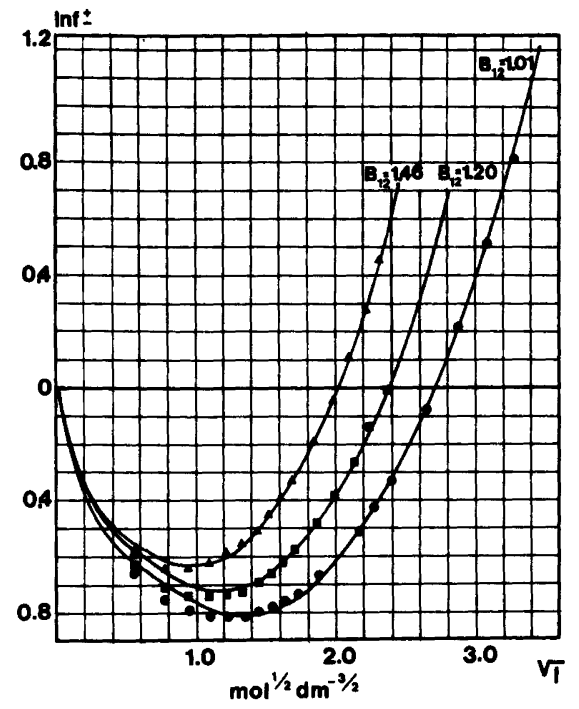

Fig. 7. In $f_{ \pm}$vs. $\sqrt{I}$ for $\operatorname{SrCl}_{2}(\Theta), \operatorname{SrBr}_{2}(\boldsymbol{\square})$ and $\mathrm{SrI}_{2}$ (А). Full lines: ASPEV.

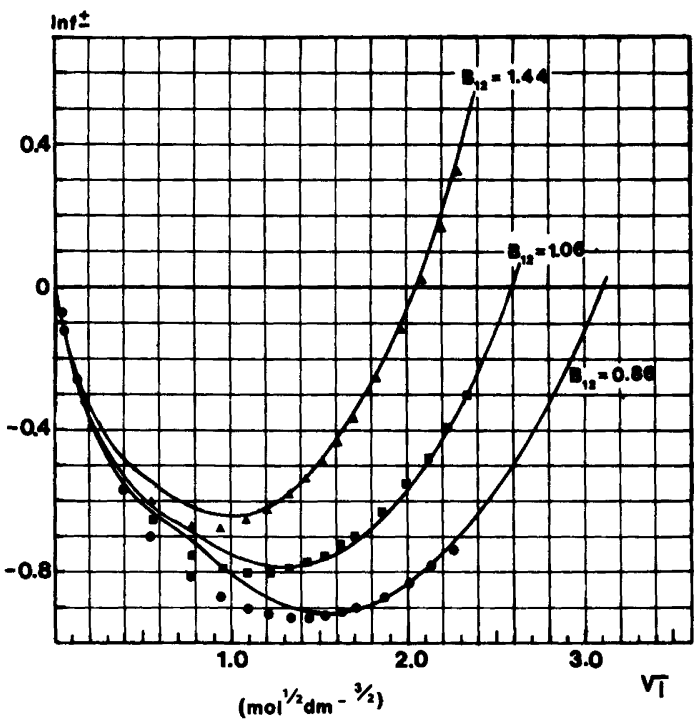

Fig. 8. In $f_{ \pm}$vs. $\sqrt{I}$ for $\mathrm{BaCl}_{2}(\bigcirc), \mathrm{BaBr}_{2}(\square)$ and $\mathrm{BaI}_{2}$ (А). Full lines: ASPEV.

values of $B_{12}$ were readjusted, see Table 2 , 3rd column. Still, there may be some maladjustments around the minimal activity coefficient and in the concentration range just to the left of the minimum. Those minor deviations seem inevitable for the following reasons: (1) It was not possible to fit the $x$-position of the minimum simultaneously with $\left(\ln y_{ \pm}\right)_{\min }, x_{\frac{1}{t}}$ and $x_{0}$. (2) The exponential concentration dependence $\exp \left(-18 B_{12} c\right)$ in the transition range is merely a guessed empirical dependence which is probably not completely representative for the true concentration dependence in this range. (3) The factor 18 in the argument of the exponential function was introduced mostly par force to cope with the mean behaviour found for the alkali halogenides in the previous paper. Better values could have been found for the two measured examples here $\left(\mathrm{CaCl}_{2}\right.$ and $\mathrm{BaCl}_{2}$, vide Figs. 3 and 4 ), but at the expense of less simplicity of the final formulae.

\section{CALCULATION OF EXCLUDED VOLUMES AND CATIONIC RADII}

For an electrolyte dissociating into $v_{+}$cations and $v_{-}$anions we have for the mean ionic activity coefficient

$\ln f_{ \pm}=-A^{*} \sqrt{I}+$

$\frac{c}{v_{+}+v_{-}}\left\{B_{11} v_{+}^{2}+2 B_{12} v_{+} v_{-}+B_{22} v_{-}^{2}\right\}$

according to the virial expansion of the osmotic pressure, see Ref. 1, eqn. (24). The coefficients $B_{11}$, $B_{12}$ and $B_{22}$ are molar excluded volume between cations, between cations and anions and between anions and anions, respectively. According to the Bronsted principle of specific interaction of ions interpreted in a quantitative way we have

$B_{11}=B_{22}=0$

The explanation may be, that ions of the same sign never come so close to each other - due to repulsive forces - that any excluded volume is "felt". As $I=\mu c$ with

$\mu=\frac{1}{2}\left\{z_{+}^{2} v_{+}+z_{-}^{2} v_{-}\right\}$

we obtain the formula

$\ln f_{ \pm}=-A^{*} \sqrt{I}+\frac{2 B_{12} v_{+} v_{-}}{\mu\left(v_{+}+v_{-}\right)} I$

By comparison with (4) it is seen that

Acta Chem. Scand. A 33 (1979) No. 8 
$B^{*}=\frac{2 v_{+} v_{-}}{\mu\left(v_{+}+v_{-}\right)} B_{12}$

In Ref. 1, eqn. (67) this formula was erroneously given with the $\mu$-factor in the numerator instead of the denominator. This error is of no consequences in the case of alkali halogenides, however, since $\mu=1$ in that case. For the earth alkali halogenides and other $2: 1$ or $1: 2$ salts we have

$B^{*}=\frac{4}{9} B_{12}$

The sum of the radii of the cation and the anion is given by

$B_{12}=\frac{4 \pi N_{0}}{3}\left(R_{+}+R_{-}\right)^{3}$

$\left(N_{0}=\right.$ Avogadro's number $)$

In Table 2 those distances of closest apprach are given as calculated from the adjusted $B_{12}$-values. If the radii of the anions are assumed to be the crystal ionic radii or Pauling radii $\left(R_{\mathrm{p}}\right)$, since they are assumed unhydrated, the ASPEV-radii of the cations can be calculated. Crystal ionic radii are taken from the tabulation in Ref. 8, page F-152. There is a certain uncertainty in using those radii, since they usually depend slightly on the kind of crystal studied or the way the quantum-mechanical calculations are made. The tabulated values are some suitable "average values". The cationic radii calculated in Table 2 are quite consistent among the salts, however, and in the next section several reasons will be given for the correctness of those radii from the point of view of ionic conductivity and ion-dipole interaction theory between the cations and the surrounding water molecules.

\section{DISCUSSION}

Fig. 9 shows that the ASPEV radii of the cations with the smallest Pauling radii are the largest. That

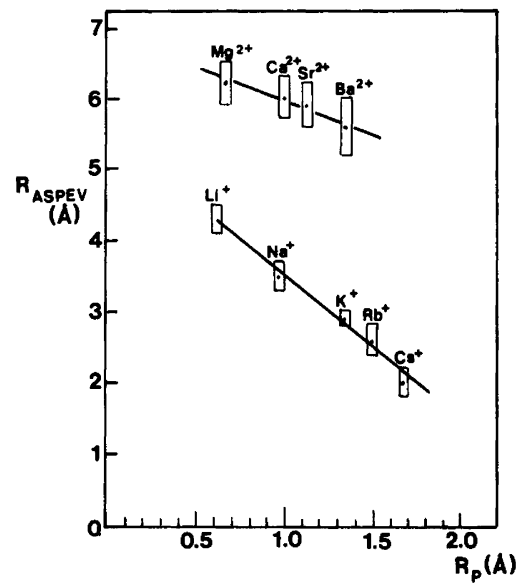

Fig. 9. The ASPEV radii $v$ s. the Pauling radii $\left(R_{\mathrm{p}}\right)$ for hydrated alkali metal and earth alkali metal cations $\left(25^{\circ} \mathrm{C}\right)$.

Table 2. Excluded volumes and radii of the earth alkali metal ions.

\begin{tabular}{llllll}
\hline Electrolyte & $\begin{array}{l}B_{12} \text { (opt) } \\
\mathrm{dm}^{3} / \mathrm{mol}\end{array}$ & $\begin{array}{l}B_{12} \text { (adjusted) } \\
\mathrm{dm}^{3} / \mathrm{mol}\end{array}$ & $\begin{array}{l}R_{+}+R_{-} \\
\AA\end{array}$ & $\begin{array}{l}R_{-} \\
\AA\end{array}$ & $\begin{array}{l}R_{+} \\
\AA\end{array}$ \\
\hline $\mathrm{MgCl}_{2}$ & 1.24 & 1.24 & 7.90 & 1.80 & \\
$\mathrm{MgBr}_{2}$ & 1.53 & 1.45 & 8.32 & 1.95 & $6.4 \pm 0.3$ \\
$\mathrm{MgI}_{2}$ & 1.71 & 1.71 & 8.79 & 2.16 & \\
$\mathrm{CaCl}_{2}$ & 1.08 & 1.08 & 7.54 & 1.80 & \\
$\mathrm{CaBr}_{2}$ & 1.35 & 1.30 & 8.02 & 1.95 & $6.0 \pm 0.3$ \\
$\mathrm{CaI}_{2}$ & 1.55 & 1.55 & 8.51 & 2.16 & \\
& & & & & \\
$\mathrm{SrCl}_{2}$ & 1.01 & 1.01 & 7.37 & 1.80 & $5.9 \pm 0.3$ \\
$\mathrm{SrBr}_{2}$ & 1.21 & 1.20 & 7.81 & 1.95 & \\
$\mathrm{SrI}_{2}$ & 1.46 & 1.46 & 8.34 & 2.16 & \\
$\mathrm{BaCl}_{2}$ & & & & & \\
$\mathrm{BaBr}_{2}$ & 0.88 & 0.86 & 6.99 & 1.80 & \\
$\mathrm{BaI}_{2}$ & 1.04 & 1.06 & 7.49 & 1.95 & \\
\hline
\end{tabular}

Acta Chem. Scand. A 33 (1979) No. 8 


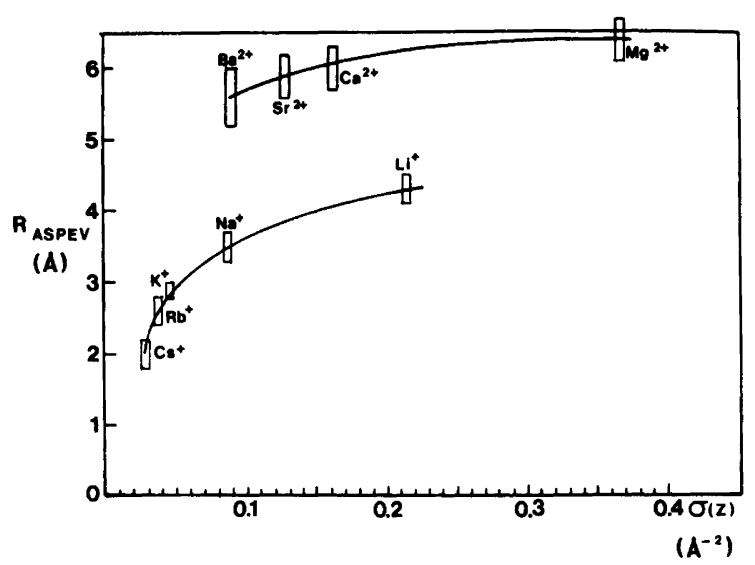

Fig. 10. The ASPEV radii of hydrated mono- and divalent cations vs. charge density on "surface" of unhydrated ions.

means probably that those ions are the most hydrated. Also, the earth alkali cations seem to be more hydrated than the alkali cations with similar radii. Looking for an explanation for that fact, one can hardly avoid noticing the significance of the surface charge of the ion. If the cation is not too small, the oxygen atoms of the water molecules will penetrate to about the Pauling radius of the ion. The ability to keep a hold on the water dipoles will be dependent on the electric field strength at the surface of the ion, which again is proportional to the surface charge density. Defining this quantity as

$\sigma(z) \equiv z / 4 \pi R_{\mathrm{p}}^{2}$

we might now make a plot of the ASPEV radii of the hydrated cations $v s$. the charge density $\sigma(z)$. This is done in Fig. 10. However, it is seen that the alkali metal ions and the earth alkali ions are still positioned on two disconnected curves.

A consistent way of plotting is obtained by the following arguments: Since the field strength at the surface of the cation is proportional to the charge density, the field strength at any distance around the ion will also be proportional to the charge density. At any distance there will be a competition between rotatory Brownian motion of the water dipoles and the unidirecting force on the dipoles by the radial electric field strength. The dependence will typically be of the well-known Langevin type (or the quantum mechanical extension of the Langevin relation). With a given unidirec- tion of the dipoles at a given position, the interaction of the dipoles with the central ion will be dependent on the product of the radial derivative of the field strength with the local polarization. Since the field strength, its derivative and the polarization are all proportional the charge $z_{+}$, it is natural to use the density of the squared charge instead of the charge density as a scaling parameter. Defining this density as

$\sigma\left(z^{2}\right) \equiv z^{2} / 4 \pi R_{\mathrm{p}}^{2}$

I have plotted the ASPEV radii $v$ s. $\sigma\left(z^{2}\right)$ in Fig. 11. Even if two separate curves are still discernible for the alkali and the earth alkali cations, it is obvious that the cations are much closer to a common curve in Fig. 11 than the case is in Figs. 9 or 10. The hydration seems to saturate at high values of the surface density of squared charge. This saturation effect may be due either to the fact that polarization of the water dipoles against the Brownian motion saturates, or to the fact that an increased surface charge does not help when the cations become smaller than the "holes" in the "closed packed lattice" of the water molecules. Even if the field strength at the surface increases, there is evidently no water to polarize before we come to the boundary of the hole. I hope to be able to give a detailed theoretical explanation for the curve in Fig. 11 in a later publication.

Finally, the consistency of the ionic radii is also checked by means of data for equivalent ionic conductivities at infinite dilution $\left(\lambda_{i}^{0}\right)$. It was sug- 


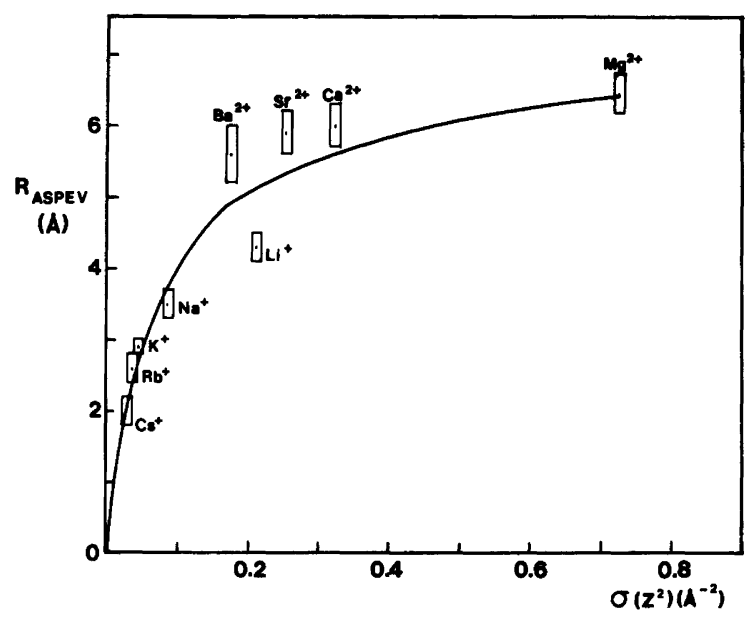

Fig. 11. The ASPEV radii of hydrated mono- and divalent cations vs. density of squared charge on unhydrated ions.

gested in Ref. 1 to correlate such data with the ionic radii by means of a modified Born-Fuoss-BoydZwanzig (mod. BFBZ) theory of viscosity and dielectric relaxation drag, i.e. an equation of the form

$\frac{\lambda_{\mathrm{i}}^{0}}{\left|z_{\mathrm{i}}\right|}=\frac{A R^{3}}{C+R^{4}}$

with $A$ and $C$ being adjustable parameters corresponding to the "Stokes' law" drag and the drag from relaxation of the dipoles, when the ion moves.
In Fig. 12 it is shown that the conductivity data can be positioned on smooth curves of the mod. BFBZ-type with the ASPEV radii as scaling parameter. Any inconsistency in our statistical mechanical calculations of ionic radii would have shown up as a discontinuity between the data for the alkali metal ions and the earth alkali ions. The uncertainty in the determination of the relaxation parameter is too great for any sensible use of that parameter, but the $A$ parameter is between 175 and 200 (in units corresponding to $R$ in $\AA$ and $\lambda_{i}^{0}$ in $\Omega^{-1} \mathrm{~cm}^{2}$ (eqv.). The theoretical Stokes' law value

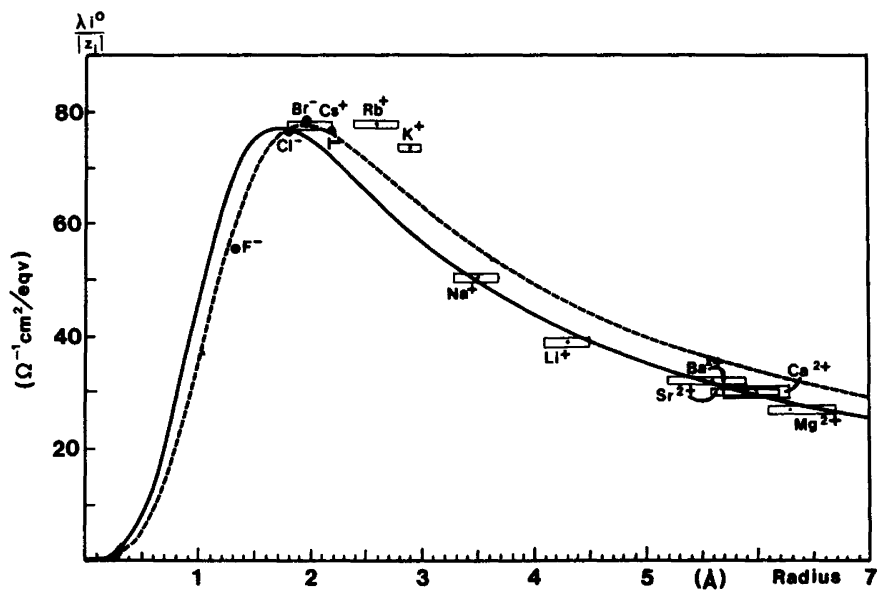

Fig. 12. Equivalent conductivities at infinite dilution for mono- and divalent cations vs. ASPEV radii. Full line: Modified BFBZ-theory $A=175, C=2.8$. Dashed line: $A=200, C=4.7$. 
of $A$ is 91.7 at $25{ }^{\circ} \mathrm{C}$, however. Since $A$ is inversely proportional to the factor $6 \pi \eta$ if Stokes law is valid, the found parameter value of 175 to 200 corresponds to a modified Stokes law friction factor of the form

Friction factor $=k \pi \eta R$

with $k$ in the range 2.8 to 3.1 . In this conection it should be mentioned that the factor 6 in the Stokes' law is valid only for a coefficient of sliding friction equal to infinity at the surface of the sphere (no slip and no sliding of the fluid at the spherical surface). For a coefficient of sliding friction equal to zero, the factor is 4 instead of 6 , and for intermediary values, the factor is between 4 and 6 , see Ref. 9. For spheres of atomic dimensions, the usual no-slip, no-sliding condition cannot be expected to hold, but the Stokes' factor seems here to be even lower than 4 . However, the deviation from Stokes' law is in the right direction, and a more quantitative correspondence is not to be expected when using macroscopic models on systems of atomic magnitude.

\section{REFERENCES}

1. Sørensen, T. S. Acta Chem. Scand. A 32 (1978) 571.

2. Brønsted, J. N. The Principle of the Specific Interaction of Ions, K. Dan. Vidensk. Selsk., Mat.-Fys. Medd. IV (1921) 4.

3. Newman, J. S. Electrochemical Systems, PrenticeHall, Englewood Cliffs, N.J. 1973, p. 94, Section 31.

4. Latimer, W. M. Oxidation Potentials, 2nd Ed., Prentice-Hall, Englewood Cliffs, N.J. 1952, Appendix II.

5. Harned, H. S. and Owen, B. B. The Physical Chemistry of Electrolyte Solutions, 3rd Ed., Van Nostrand-Reinold, New York 1958, Appendix A.

6. Robinson, R. A. and Stokes, R. H. Electrolyte Solutions, 2nd Ed., Butterworths, London 1959, App. 8.10.

7. Taschenbuch für Chemiker und Physiker, 3. Auflage, Band I, Lax, E. Ed., Springer, BerlinHeidelberg-New York 1967, Tafel 32212, pp. $809-814$.

8. Handbook of Chemistry and Physics, 50th Ed., Weast, R. C. Ed., The Chemical Rubber Co., $1969-1970, \mathrm{D}-175$.

9. Lamb, H. Hydrodynamics, Dover, New York $1945, \$ 337$.

Received March 26, 1979. 